\title{
Using reported pathogenic variants to identify therapeutic opportunities for genetic diseases
}

\author{
Andrew Ressler ${ }^{1}$ and David Goldstein ${ }^{2}$ \\ ${ }^{1}$ Columbia University Medical Center \\ ${ }^{2}$ Columbia Institute for Genomic Medicine
}

August 25, 2020

\begin{abstract}
Purpose Drug development strategies for genetic diseases depend critically on accurate knowledge of how pathogenic variants cause disease. For some well-studied genes, the direct effects of pathogenic variants are well documented as loss of function, gain of function or hypermorphic, or a combination of the two. For many genes, however, even the direction of effect of variants remains unclear. Classification of Mendelian disease genes in terms of whether pathogenic variants are loss or gain of function would directly inform drug development strategies. Methods We leveraged the recent dramatic increase in reported pathogenic variants to provide a novel approach to inferring the direction of effect of pathogenic variants. Specifically, we quantify the ratio of reported pathogenic variants that are missense compared to loss of function. Results We first show that for many genes that cause dominant Mendelian disease, the ratio of reported pathogenic missense variants is diagnostic of whether the gene causes disease through loss or gain of function, or a combination. Second, we identify a set of genes that appear to cause disease largely or entirely through gain of function or hypermorphic pathogenic variants. Conclusions We suggest a set of 16 genes suitable for drug developmental efforts utilizing direct inhibition.
\end{abstract}

\section{Keywords:}

Gain-of-function, drug development, missense variants, therapeutic inhibition, autosomal dominant

\section{Introduction}

Determining whether a genetic disorder is due to a gain, loss or change of protein function is a critical first step in effective drug discovery. For many recessive disease genes, including many inborn errors of metabolism, pathogenic variants have been clearly identified as loss of function. Similarly, for a number of dominant disease genes, careful functional characterization of variants found in patients has provided clear evidence of variantal effects. For example, dominant pathogenic variants in the NSD1 gene have been shown to reduce or eliminate the function of $\mathrm{NSD}^{1,2}$, whereas nearly all apparently pathogenic variants in SCN8A and KCNT1 have been clearly shown to be variants that increase channel current ${ }^{3,4,5,6}$. Furthermore, some disease genes have been clearly shown to carry both pathogenic gain of function and loss of function variants. For example, after the identification of loss of SMCHD1 function is causative for a form of muscular dystrophy $^{7}$, later research identified gain-of-function variants in SMCHD1 as responsible for rare syndrome $\mathrm{BAMS}^{8}$, a distinct genetic disease. Overall, although many recessive genes are classified as due to loss of function (LoF) variants, and a subset of dominant genes are classified as haploinsufficient, meaning that disease is due to loss of activity of one of the two alleles, many dominant genes remain not clearly classifiable as due to either loss or gain of function variants. The secure identification of which of these unclassified genes cause disease because of variants that increase or change the activity of the encoded protein would have immediate implications for drug development. 
Over the past decade, a wide range of approaches have been used to infer the functional impact of pathogenic variants $^{9,10,11}$. Attempts to identify $\mathrm{LoF}$ and GoF variants have leveraged existing bioinformatic tools like genetic tolerance sorting (SIFT), polymorphism phenotyping (PolyPhen) ${ }^{12}$ and conservation based Hidden Markov Models ${ }^{13}$. Additionally, highly supervised approaches that manually examine variants suggested to be GoF within OMIM have been attempted ${ }^{14}$. Despite these advances, identifying a subset of genetic diseases well suited for therapeutic inhibition has yet to be well established. Surprisingly, no one has yet attempted to use the distribution of reported pathogenic variants to infer whether pathogenic variants are strictly gain or loss of function, or some combination. The intuition behind this approach is straightforward. Genome wide it has been estimated that approximately $20 \%$ of missense variants are significant hypermorphic or loss of function variants ${ }^{15}$. In addition, on average, the proportion of variants in a human gene that are missense versus nonsense variants has been estimated to be about $1.05^{16}$. This means that for genes that cause disease due to haploinsufficiency, the proportion of pathogenic missense to all missense and LoF variants should be approximately 0.21 . This intuition is clearly supported by considering the well-known examples of NSD1 and KCNT1. Of all reported pathogenic (mostly de novo) variants in NSD1, the proportion of missense variants is 0.27 (88 missense, $242 \mathrm{LoF}$ ), whereas KCNT1 has 38 reported pathogenic missense variants and no known pathogenic LoF variants. Based on this intuition, we have developed an evaluation of the proportion of variant type in all autosomal dominant genes in order to infer the direction of effect of pathogenic variants. Specifically, we seek to find a threshold on the proportion of variants that are missense versus LoF that is diagnostic of whether the gene causes disease due to loss or gain of function / hypermorphism. For convenience, hereafter, we will refer to both the gain of function and hypermorphism as "gain of function" (GoF), without attempting to distinguish between the two.

\section{Methods}

To develop a pipeline to distinguish genes that cause disease due to $\mathrm{LoF}$ or GoF, we first extracted all pathogenic and likely pathogenic variants from ClinVar's GRCh37 weekly VCF file with minor allele frequencies of 0 in all three of Exac, GO-ESP and GMAF (figure 1). We hypothesized Autosomal Dominant variants will predominantly cause disease via haploinsufficiency or GoF. Thus, we focused our analyses on known Haploinsufficient genes ( $\mathrm{n}=361)$ and OMIM annotated autosomal dominant ("AD") genes ( $\mathrm{n}=219)$.

We then categorized variants as "likely LoF" if they were annotated as "nonsense", "frame-shift" or "stoploss" and as "missense" if they were annotated as "missense." All other variant types were not binned as either missense or likely LoF variants and were not include in ratio calculations. On the occasions where the same variant was annotated as both "likely LoF" and "missense," the variant was excluded from downstream analyses.

To assess whether the variant ratio is generally diagnostic of how variants caused disease, we first considered a set of genes that have been defined previously as haploinsufficient. To this end, we leveraged two separately generated lists of genes. First, we considered a list of genes ("Dang") generated through Dang et al.'s robust database-mining of OMIM and PubMed ${ }^{17}$. We additionally considered ClinGen's manually curated and reviewed list of 312 genes ("ClinGen") determined to have "sufficient evidence of haploinsufficiency." Out of these gene lists, a total of 361 unique haploinsufficient ("HI") genes had more than $10 \mathrm{P} / \mathrm{LP}$ variants. Of these 361 entries, 93 were shared, 63 were unique to Dang and 205 were unique to ClinGen. We considered both lists in order to identify a threshold on the variant ratio for autosomal dominant genes not annotated as haploinsufficient. Noting that genes that cause more than one Mendelian disease can have different directions of effects for different diseases, we also separately distinguished genes responsible for only one Mendelian disease.

\section{Results}

\section{Autosomal Dominant Genes Enriched for Missense Variants}

In all genes considered ( $\mathrm{HI}=361$, OMIM autosomal dominant, 'AD', = 219), we identified pathogenic or likely pathogenic variants classified as either missense or LoF (figure 1). We first evaluated the ratio of missense to all pathogenic variants for HI genes that are associated only with a single Mendelian disease 
(figure 1). We found that for known HI genes associated only with a single Mendelian disease, $95 \%$ of all $\mathrm{HI}$ genes have a missense ratio less than $0.8(128 / 135)$ and the median missense ratio for all haploinsufficient genes is 0.22 , nearly identical to the a priori predicted ratio of missense variants. Importantly, since the generation of Dang's list of HI genes, more recent research has clearly demonstrated haploinsufficiency is not the predominant mechanism of disease for variants of, MYOC and SH3BP2, while additional pathogenic GoF or dominant-negative variants have been identified in KCNQ4 and SLC40A1 $1^{18,19,20,21,22}$. Further, the three HI genes from ClinGen surpassing a threshold of 0.8 (OTC,PGK1,SMS) are all found on the X-chromosome. Importantly, a simple threshold may identify more false positives when the total number of variants is lower. Thus, we alternatively considered the lower bound of a $95 \%$ binomial confidence interval and did not find a significant enhancement of signal (figure S1). Given similar results when considering a binomial lower bound and the successful exclusion of haploinsufficiency, a simple threshold is sufficient to exclude haploinsufficiency as a likely mechanism for $\mathrm{AD}$ genes.

Based on this finding, we considered all AD genes not known to be HI that are associated with only a single Mendelian disease, and we find 51 out of 110 applicable AD genes that appear to cause disease through GoF. Among this set of genes with variant ratios indicative of GoF, we find genes well known to cause disease due to GoF, such as GFAP and RIT1 (figure 2a).

We then investigated whether or not our HI- threshold could be extended to all genes with at least one disease annotated as AD, including those that cause multiple Mendelian conditions. We found similar enrichment of $\mathrm{AD}$ genes and absence of $\mathrm{HI}$ genes above our threshold (figure $2 \mathrm{~b}$ ). Using our HI- threshold and more permissive inclusion criteria, we generated a list of $121 \mathrm{AD}$ genes (table S1) likely to act through a gain-offunction. Importantly, we find the presence of aforementioned known GoF genes such a KCNT1 and SCN8A within this gene list.

Finally, we sought to examine the topological distribution of missense variants in GoF AD genes, given GoF variants in the aggregate tend to be more spatially clustered ${ }^{23}$. As hypothesized, AD genes tended to be more clustered than known HI genes and autosomal recessive OMIM genes (figures S2). However, the distributions of clustering were overlapping and a clear way to incorporate clustering to complement a simple missense threshold was not apparent.

\section{Identifying GoF Genes for Drug Targeting}

Once we generated a threshold capable of reliably identifying likely GoF genes, we aimed to determine a subset of genes well suited for therapeutic inhibition. To assess whether inhibition is likely to be well tolerated, we considered whether the genes are under strong selection against loss of function variants. To this end, we only considered GoF genes highly tolerant to loss of function variants ${ }^{24}(\mathrm{pLI}<0.1)$.

When considering all $\mathrm{AD}$ and $\mathrm{HI}$ genes, lower $\mathrm{pLI}$ scores are correlated with increasing ratio of missense variants. However, the strength of correlation is minimal. Further, the distributions of AD genes and HI are not cleanly distinguished and 11 of the 35 genes with $\mathrm{pLI}<0.1$ are known $\mathrm{HI}$ genes, including two genes, PKD2 and TRAPPC2, that are found in both HI sources (figure 3). Thus, the addition of pLI is not redundant and complementary to our missense ratio threshold.

Amongst the $\mathrm{AD}$ genes that appear to act through a GoF based on missense ratio, we identified 36 that show no evidence of strong selection against loss of function variants in the human population. Finally, we manually cross-referenced our list with "The Drug Gene Interaction Database" 25 to identify a set of genes known to be therapeutically accessible. Following curation, we identified a list of 16 druggable, LoF tolerant, likely GoF genes (table 1).

\section{Discussion}

Identifying causal GoF disease genes tolerant of reduced dosage would provide therapeutic targets of immediate interest. Further, publicly available drugs are more often inhibitors than activators, suggesting enhanced therapeutic potential for downregulation ${ }^{26}$. Identifying likely GoF genes has proved relatively difficult, as displayed by the distribution of pLI scores for known Haploinsufficient genes and significant reduction in 
performance of Polyphen and SIFT compared to prediction of LoF variants ${ }^{12}$. Despite these difficulties, several groups have developed methods to identify likely GoF variants, but a definitive list of GoF genes remains elusive. Here, we leveraged the increasing number of known pathogenic / likely pathogenic variants to generate a HI- threshold that identifies likely GoF genes. We further parsed these likely GoF genes to identify a subset of targets that were both therapeutically accessible and LoF tolerant.

Well characterized GoF genes, such as SCN8A, SOS1 and KCNT1 are present in the list of likely GoF genes, alongside mischaracterized "known haploinsufficient genes" like MYOC and SH3BP2. However, these genes all have been relatively robustly assessed in vitro, while many pathogenic variants have very limited functional evidence in the literature and can benefit particularly from a hypothesis on functional mechanism. Further, our list of likely GoF genes with low pLIs includes GFAP, which when targeted with antisense inhibition, has shown the potential benefit of utilizing drug inhibition on candidate genes ${ }^{27}$.

Importantly, within our analyses, we did not attempt to distinguish between hypermorphic variants and other GoF mechanisms. Similarly, we did not consider whether or not a variant may act through a dominant negative mechanism. Such genes may be present within our GoF list and additional strategies would be required to confidently exclude them.

Finally, as publicly available datasets continue to increase in size, the list of genes with more than 10 variants that surpass HI- threshold will continue to increase. Thus, the list of therapeutically accessible likely GoF genes will expand and may provide important context when considering which treatment candidate to prioritize in vitro when investigating novel causal variants. Further, recent work from our lab and others has leveraged published RNA sequencing data to identify downregulators of gene targets ${ }^{28,29}$. A similar approach in this context would be complementary and may lead to rapid successful drug repurposing capable of providing direct benefit to patients.

\section{Conflict of Interest Statement:}

David Goldstein holds equity in Praxis Medicines

\section{Data Availability:}

The data that supports the work within this manuscript is publicly available on ClinVar's weekly GRCh37 weekly vcf file (https://ftp.ncbi.nlm.nih.gov/pub/clinvar/vcf_GRCh37/weekly/) and through OMIM's data downloads ${ }^{31}$ (https://omim.org/downloads/???/mimTitles.txt). The work within this manuscript is up to date as of August $3^{\text {rd }}, 2021$.

\section{References}

1. Choufani, S., Cytrynbaum, C., Chung, B. H. Y., Turinsky, A. L., Grafodatskaya, D., Chen, Y. A., Cohen, A. S. A., Dupuis, L., Butcher, D. T., Siu, M. T., Luk, H. M., Lo, I. F. M., Lam, S. T. S., Caluseriu, O., Stavropoulos, D. J., Reardon, W., Mendoza-Londono, R., Brudno, M., Gibson, W. T., ... Weksberg, R. (2015). NSD1 mutations generate a genome-wide DNA methylation signature. Nature Communications ,6 , 10207.https://doi.org/10.1038/ncomms10207

2. McClelland, J., Burgess, B., Crock, P., \& Goel, H. (2016). Sotos syndrome: An unusual presentation with intrauterine growth restriction, generalized lymphedema, and intention tremor.American Journal of Medical Genetics Part A , 170 (4), 1064-1069.https://doi.org/10.1002/ajmg.a.37535

3. Pan, Y., \& Cummins, T. R. (2020). Distinct functional alterations in SCN8A epilepsy mutant channels. The Journal of Physiology ,598 (2), 381-401.https://doi.org/10.1113/JP278952

4. Lopez-Santiago, L. F., Yuan, Y., Wagnon, J. L., Hull, J. M., Frasier, C. R., O'Malley, H. A., Meisler, M. H., \& Isom, L. L. (2017). Neuronal hyperexcitability in a mouse model of SCN8A epileptic encephalopathy. Proceedings of the National Academy of Sciences , 114 (9), 23832388.https://doi.org/10.1073/pnas.1616821114

5. Milligan, C. J., Li, M., Gazina, E. V., Heron, S. E., Nair, U., Trager, C., Reid, C. A., Venkat, A., Younkin, D. P., Dlugos, D. J., Petrovski, S., Goldstein, D. B., Dibbens, L. M., Scheffer, I. E., Berkovic, 
S. F., \& Petrou, S. (2014). KCNT1 gain of function in 2 epilepsy phenotypes is reversed by quinidine. Annals of Neurology, 75 (4), 581-590.https://doi.org/10.1002/ana.24128

6. Quraishi, I. H., Stern, S., Mangan, K. P., Zhang, Y., Ali, S. R., Mercier, M. R., Marchetto, M. C., McLachlan, M. J., Jones, E. M., Gage, F. H., \& Kaczmarek, L. K. (2019). An Epilepsy-Associated KCNT1 Mutation Enhances Excitability of Human iPSC-Derived Neurons by Increasing Slack K $\mathrm{Na}$ Currents. The Journal of Neuroscience , 39 (37), 74387449.https://doi.org/10.1523/JNEUROSCI.1628-18.2019

7. Lemmers, R. J. L. F., Tawil, R., Petek, L. M., Balog, J., Block, G. J., Santen, G. W. E., Amell, A. M., van der Vliet, P. J., Almomani, R., Straasheijm, K. R., Krom, Y. D., Klooster, R., Sun, Y., den Dunnen, J. T., Helmer, Q., Donlin-Smith, C. M., Padberg, G. W., van Engelen, B. G. M., de Greef, J. C., ... van der Maarel, S. M. (2012). Digenic inheritance of an SMCHD1 mutation and an FSHD-permissive D4Z4 allele causes facioscapulohumeral muscular dystrophy type 2. Nature Genetics , 44 (12), 1370-1374.https://doi.org/10.1038/ng.2454

8. Gurzau, A. D., Chen, K., Xue, S., Dai, W., Lucet, I. S., Ly, T. T. N., Reversade, B., Blewitt, M. E., \& Murphy, J. M. (2018). FSHD2- and BAMS-associated mutations confer opposing effects on SMCHD1 function.Journal of Biological Chemistry , 293 (25), 98419853.https://doi.org/10.1074/jbc.RA118.003104

9. Dong, C., Wei, P., Jian, X., Gibbs, R., Boerwinkle, E., Wang, K., \& Liu, X. (2014). Comparison and integration of deleteriousness prediction methods for nonsynonymous SNVs in whole exome sequencing studies. Human Molecular Genetics , 24 (8), 2125-2137.https://doi.org/10.1093/hmg/ddu733

10. Liu, M., Watson, L. T., \& Zhang, L. (2015). HMMvar-func: A new method for predicting the functional outcome of genetic variants. BMC Bioinformatics , 16 .https://doi.org/10.1186/s12859-015-0781-z

11. Li, Y., Zhang, Y., Li, X., Yi, S., \& Xu, J. (2019). Gain-of-Function Mutations: An Emerging Advantage for Cancer Biology. Trends in Biochemical Sciences , 44 (8), 659674.https://doi.org/10.1016/j.tibs.2019.03.009

12. Flanagan, S. E., Patch, A.-M., \& Ellard, S. (2010). Using SIFT and PolyPhen to Predict Loss-ofFunction and Gain-of-Function Mutations. Genetic Testing and Molecular Biomarkers , 14 (4), 533537.https://doi.org/10.1089/gtmb.2010.0036

13. Liu, M., Watson, L. T., \& Zhang, L. (2014). Classification of Mutations by Functional Impact Type: Gain of Function, Loss of Function, and Switch of Function. In M. Basu, Y. Pan, \& J. Wang (Eds.), Bioinformatics Research and Applications (pp. 236-242). Springer International Publishing.https://doi.org/10.1007/978-3-319-08171-7_21

14. Chen, B., \& Altman, R. B. (2017). Opportunities for developing therapies for rare genetic diseases: Focus on gain-of-function and allostery. Orphanet Journal of Rare Diseases , 12 .https://doi.org/10.1186/s13023-017-0614-4

15. Kryukov, G. V., Pennacchio, L. A., \& Sunyaev, S. R. (2007). Most Rare Missense Alleles Are Deleterious in Humans: Implications for Complex Disease and Association Studies. American Journal of Human Genetics, 80 (4), 727-739.

16. Gorlov, I. P., Kimmel, M., \& Amos, C. I. (2006). Strength of the purifying selection against different categories of the point mutations in the coding regions of the human genome. Human Molecular Genetics , 15 (7), 1143-1150.https://doi.org/10.1093/hmg/ddlo29

17. Dang, V. T., Kassahn, K. S., Marcos, A. E., \& Ragan, M. A. (2008). Identification of human haploinsufficient genes and their genomic proximity to segmental duplications. European Journal of Human Genetics , 16 (11), 1350-1357.https://doi.org/10.1038/ejhg.2008.111

18. Shepard, A. R., Jacobson, N., Millar, J. C., Pang, I.-H., Steely, H. T., Searby, C. C., Sheffield, V. C., Stone, E. M., \& Clark, A. F. (2007). Glaucoma-causing myocilin mutants require the Peroxisomal targeting signal-1 receptor (PTS1R) to elevate intraocular pressure.Human Molecular Genetics , 16 (6), 609-617.https://doi.org/10.1093/hmg/ddm001

19. Kim, B. S., Savinova, O. V., Reedy, M. V., Martin, J., Lun, Y., Gan, L., Smith, R. S., Tomarev, S. I., John, S. W. M., \& Johnson, R. L. (2001). Targeted Disruption of the Myocilin Gene (Myoc) Suggests that Human Glaucoma-Causing Mutations Are Gain of Function. Molecular and Cellular Biology , 21 
(22), 7707-7713.https://doi.org/10.1128/MCB.21.22.7707-7713.2001

20. Reichenberger, E. J., Levine, M. A., Olsen, B. R., Papadaki, M. E., \& Lietman, S. A. (2012). The role of SH3BP2 in the pathophysiology of cherubism. Orphanet Journal of Rare Diseases , 7 (Suppl 1), S5.https://doi.org/10.1186/1750-1172-7-S1-S5

21. Kamada, F., Kure, S., Kudo, T., Suzuki, Y., Oshima, T., Ichinohe, A., Kojima, K., Niihori, T., Kanno, J., Narumi, Y., Narisawa, A., Kato, K., Aoki, Y., Ikeda, K., Kobayashi, T., \& Matsubara, Y. (2006). A novel KCNQ4 one-base deletion in a large pedigree with hearing loss: Implication for the genotypephenotype correlation. Journal of Human Genetics , 51 (5), 455-460.https://doi.org/10.1007/s10038006-0384-7

22. Zhang, W., Xu, A., Li, Y., Zhao, S., Zhou, D., Wu, L., Zhang, B., Zhao, X., Wang, Y., Wang, X., Duan, W., Wang, Q., Nan, Y., You, H., Jia, J., Ou, X., Huang, J., \& China Registry of Genetic/Metabolic Liver Diseases (CR-GMLD) Group (2019). A novel SLC40A1 p.Y333H mutation with gain of function of ferroportin: A recurrent cause of haemochromatosis in China. Liver international : official journal of the International Association for the Study of the Liver , 39 (6), 1120-1127. https://doi.org/10.1111/liv.14013

23. Lelieveld, S. H., Wiel, L., Venselaar, H., Pfundt, R., Vriend, G., Veltman, J. A., Brunner, H. G., Vissers, L. E. L. M., \& Gilissen, C. (2017). Spatial Clustering of de Novo Missense Mutations Identifies Candidate Neurodevelopmental Disorder-Associated Genes. The American Journal of Human Genetics , 101 (3), 478-484.https://doi.org/10.1016/j.ajhg.2017.08.004

24. Lek, M., Karczewski, K. J., Minikel, E. V., Samocha, K. E., Banks, E., Fennell, T., O’Donnell-Luria, A. H., Ware, J. S., Hill, A. J., Cummings, B. B., Tukiainen, T., Birnbaum, D. P., Kosmicki, J. A., Duncan, L. E., Estrada, K., Zhao, F., Zou, J., Pierce-Hoffman, E., Berghout, J., .. MacArthur, D. G. (2016). Analysis of protein-coding genetic variation in 60,706 humans. Nature ,536 (7616), 285-291.https://doi.org/10.1038/nature19057

25. Cotto, K. C., Wagner, A. H., Feng, Y.-Y., Kiwala, S., Coffman, A. C., Spies, G., Wollam, A., Spies, N. C., Griffith, O. L., \& Griffith, M. (2018). DGIdb 3.0: A redesign and expansion of the drug-gene interaction database. Nucleic Acids Research , 46 (D1), D1068D1073.https://doi.org/10.1093/nar/gkx1143

26. Law, V., Knox, C., Djoumbou, Y., Jewison, T., Guo, A. C., Liu, Y., Maciejewski, A., Arndt, D., Wilson, M., Neveu, V., Tang, A., Gabriel, G., Ly, C., Adamjee, S., Dame, Z. T., Han, B., Zhou, Y., \& Wishart, D. S. (2014). DrugBank 4.0: Shedding new light on drug metabolism.Nucleic Acids Research , 42 (Database issue), D1091-D1097.https://doi.org/10.1093/nar/gkt1068

27. Hagemann, T. L., Powers, B., Mazur, C., Kim, A., Wheeler, S., Hung, G., Swayze, E., \& Messing, A. (2018). Antisense suppression of glial fibrillary acidic protein as a treatment for Alexander disease.Annals of Neurology , 83 (1), 27-39.https://doi.org/10.1002/ana.25118

28. Wang, X., Dhindsa, R., Povysil, G., Zoghbi, A., Motelow, J., Hostyk, J., Nickols, N., Rettig, M., \& Goldstein, D. B. (2020). TMPRSS2 Transcriptional Inhibition as a Therapeutic Strategy for COVID-19 .https://doi.org/10.20944/preprints202003.0360.v2

29. Shukla, R., Henkel, N. D., Alganem, K., Hamoud, A., Reigle, J., Alnafisah, R. S., Eby, H. M., Imami, A. S., Creeden, J., Miruzzi, S. A., Meller, J., \& Mccullumsmith, R. E. (2020). Integrative Omics for Informed Drug Repurposing: Targeting CNS Disorders. BioRxiv , 2020.04.24.060392.https://doi.org/10.1101/2020.04.24.060392

30. Landrum MJ, Lee JM, Benson M, Brown GR, Chao C, Chitipiralla S, Gu B, Hart J, Hoffman D, Jang W, Karapetyan K, Katz K, Liu C, Maddipatla Z, Malheiro A, McDaniel K, Ovetsky M, Riley G, Zhou G, Holmes JB, Kattman BL, Maglott DR. ClinVar: improving access to variant interpretations and supporting evidence. Nucleic Acids Res . 2018 Jan 4. PubMed PMID: 29165669 .

31. Online Mendelian Inheritance in Man, OMIM ${ }^{\circledR}$. McKusick-Nathans Institute of Genetic Medicine, Johns Hopkins University (Baltimore, MD), August $3^{\text {rd }}$, 2020. World Wide Web URL: https://omim.org/ 

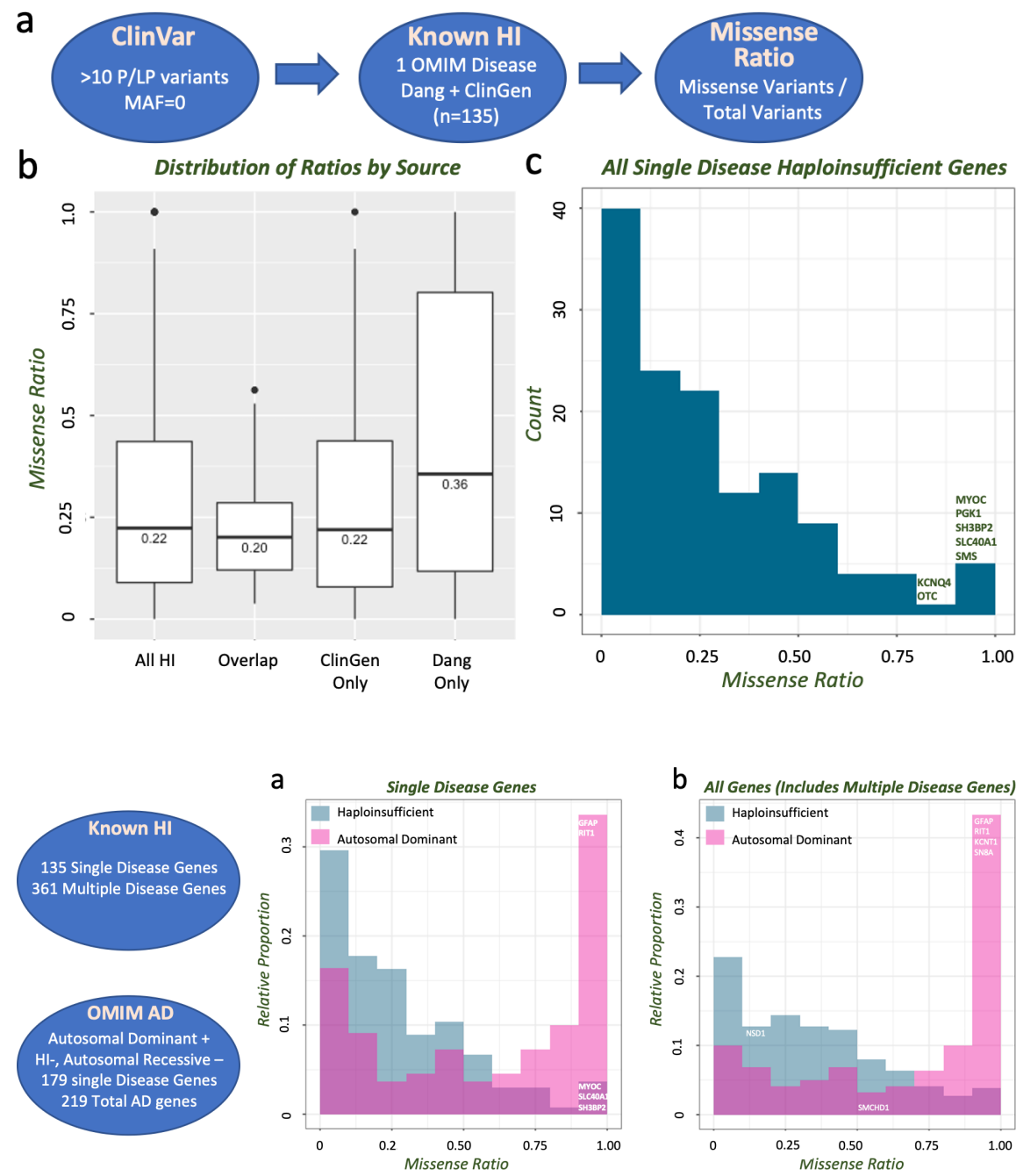

b All Genes (Includes Multiple Disease Genes)

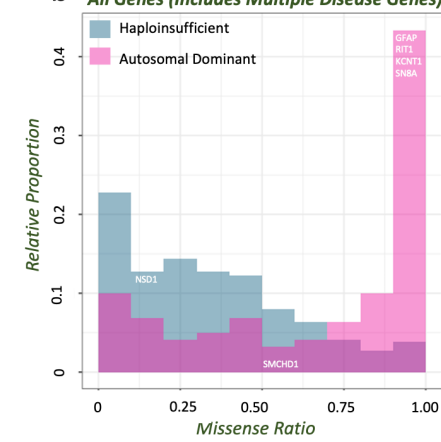

a

b Single Disease Genes

C All Genes
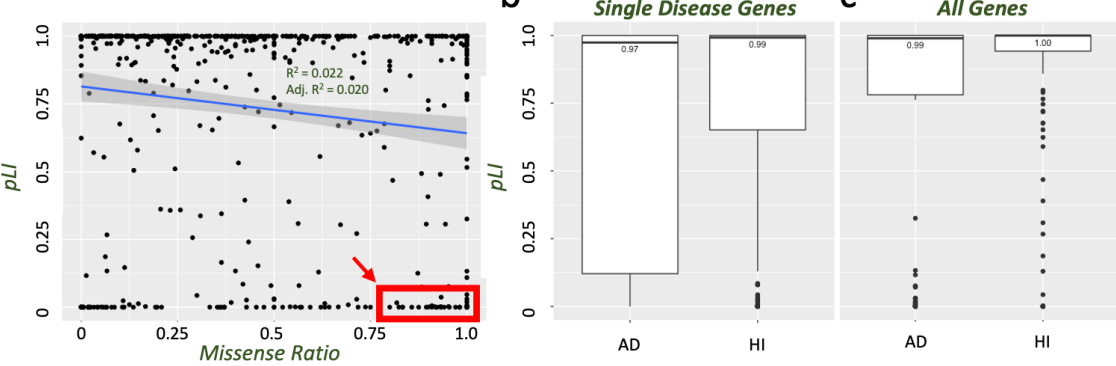

Hosted file

Table1.docx available at https://authorea.com/users/353180/articles/477219-using-reportedpathogenic-variants-to-identify-therapeutic-opportunities-for-genetic-diseases 